\title{
Cre/lox-mediated chromosomal integration of biosynthetic gene clusters for heterologous expression in Aspergillus nidulans
}

\author{
Indra Roux ${ }^{1^{*}}$, Yit-Heng Chooi ${ }^{1^{*}}$ \\ ${ }^{1}$ School of Molecular Sciences, University of Western Australia, Perth, WA 6009, Australia. \\ *Correspondence to: yitheng.chooi@uwa.edu.au or indra.roux@uwa.edu.au
}

\section{Abstract}

Building strains of filamentous fungi for stable long-term heterologous expression of large biosynthetic pathways is limited by the low transformation efficiency or genetic stability of current methods. Here, we developed a system for targeted chromosomal integration of large biosynthetic gene clusters in Aspergillus nidulans based on site-specific recombinasemediated cassette exchange. We built $A$. nidulans strains harboring a chromosomal landing pad for Cre/lox-mediated recombination and demonstrated efficient targeted integration of a $21 \mathrm{~kb}$ DNA fragment in a single step. We further evaluated the integration at two loci by analyzing the expression of a fluorescent reporter and the production of a heterologous polyketide metabolite. We compared chromosomal expression at those landing loci to episomal AMA1-based expression, which also shed light on uncharacterized aspects of episomal expression in filamentous fungi. This is the first demonstration of site-specific recombinase-mediated integration in filamentous fungi, setting the foundations for the further development of this tool.

Keywords: biosynthetic gene clusters, Cre/loxP, heterologous expression, filamentous fungi, natural products

\section{Introduction}

Filamentous fungi are prolific producers of enzymes and bioactive metabolites with biotechnological applications in pharmaceutical, agricultural and food industries. ${ }^{1,2}$ Importantly, fungal secondary metabolites (SMs) remain a promising source of novel drug leads. ${ }^{3}$ The genes required to produce a SM are usually colocalized in the genome, forming biosynthetic gene clusters (BGCs). ${ }^{3}$ Each BGC contains around 2-20 genes including a large backbone enzyme, such as a polyketide synthase (PKS) or a non-ribosomal peptide synthetase. BGCs can easily be identified in fungal genome sequences and uncharacterized BGCs represent an almost untapped resource for compound discovery. ${ }^{4}$ However, a large 
fraction of BGCs remain silent or lowly expressed under standard culture conditions, which limits their analysis. ${ }^{3}$ Additionally, as we gain access to the genomic information of diverse fungi promising BGC candidates are often identified in fungal species that are difficult to cultivate or genetically intractable. Hence, heterologous expression of BGCs in hosts with more genetic tools available has become an attractive strategy for genome-based natural product discovery from such fungi. ${ }^{5}$

Filamentous fungi hosts present several advantages for the heterologous expression of BGCs from other filamentous fungi, such as increased compatibility of promoters and intron splicing, and their natural capability for producing SMs. ${ }^{5-7}$ In particular, Aspergillus nidulans has been widely used to produce SMs by chromosomal integration or episomal expression of heterologous BGCs. ${ }^{7}$ Our laboratory has been continuing to expand the synthetic biology toolbox for $A$. nidulans, including a tripartite AMA1-based episomal vector system and CRISPRa for silent BGC activation. ${ }^{8,9}$ Episomal systems based on the replicator AMA1 have facilitated the reconstitution of BGCs for SM discovery due to their high transformation efficiency compared to integrative vectors. ${ }^{10-12}$ However, the phenotypic stability of AMA1vectors has been shown to be limiting. ${ }^{13}$ Therefore, chromosomal expression is preferred for large-scale long-term stable bioproduction in industrial settings. ${ }^{14}$ As strains with chromosomally integrated genes can be grown in low-cost complex substrates, such as agricultural by-products, ${ }^{15}$ as they do not require selection pressures to maintain heterologous genes.

Chromosomal integration of heterologous BGCs can be achieved via random or targeted integration. Given the outcomes of random integration is less predictable and extensive screening is sometimes required to identify producing strains, targeted integration is often preferred. ${ }^{16}$ Currently, targeted integration in A. nidulans is pursued through homologous recombination $(\mathrm{HR})$ facilitated by strains deficient in the non-homologous end joining pathway $(\Delta n k u A) .{ }^{17}$ However, HR efficiency drops when integrating constructs larger than a few kilobases. ${ }^{14}$ As a result, HR-mediated integration of large BGCs relies on the laborious sequential integration of smaller BGC fragments. ${ }^{18,19}$ The efficiency of HR can be increased by creating a CRISPR/Cas induced double stranded DNA (dsDNA) break, but multiple integration rounds may still be needed to reconstitute a full cluster. ${ }^{20-22}$ To leverage the increasing amount of BGCs identified in novel fungi, new methods are needed for the effective one-step creation of strains for heterologous BGC expression. ${ }^{4}$

Here, we develop a Cre/lox site-specific recombinase system for one-step chromosomal integration of BGCs in the heterologous host $A$. nidulans. Site-specific recombinases are well suited for the integration of large DNA regions, as they mediate the strand exchange between the recombination sites in a size-independent manner and orthogonally to the host 
machinery. ${ }^{23}$ As Cre/loxP recombination is reversible, strategies for irreversible integration rely on 34-bp lox sites with mutations either in the 8-bp asymmetric core or 13-bp palindromic ends. Heteromeric lox sites contain nucleotide variations in the left or right ends, respectively named LE and RE (Figure 1, Table S1). ${ }^{24}$ Recombination between LE/RE mutant lox sites on a chromosomal location and a donor vector will result in a stable integration as the resulting Iox site on the chromosome will contain both LE and RE mutations and can no longer recombine. ${ }^{23}$ However, on its own LE/RE integration results in the insertion of the whole donor vector (Figure 1). A cleaner strategy for integration relies on two sequential recombination events between two pairs of heterospecific lox sites (harboring mutations in the core region) named Recombinase Mediated Cassette Exchange (RMCE). In RMCE the chromosomal landing pad is flanked by two mutually incompatible lox sites that can recombine with corresponding compatible sites located in the donor vector flanking the genes of interest (Figure 1). In the presence of the Cre recombinase, the donor vector is first integrated via recombination with one of the lox sites at the landing pad. Subsequently, a second recombination event takes place chromosomally between two compatible lox sites, resulting in the excision of the donor vector backbone and the original landing pad cassette, leaving behind the genes of interest irreversibly integrated (Figure 1). RMCE has been used for the integration of large constructs in a wide range of cell factories and model organisms. ${ }^{25-29}$

Although Cre/lox-mediated deletion has been used to recycle marker genes in filamentous fungi, ${ }^{30-37}$ recombinase-mediated chromosomal integration remained unadopted. Here, we build a vector set for Cre/lox-mediated integration in filamentous fungi and demonstrate that this tool is an efficient alternative for the integration of large heterologous BGCs in A. nidulans. As a proof-of-concept, we integrate a $21.4 \mathrm{~kb}$ DNA fragment containing six genes, including a large polyketide synthase gene, for the heterologous production of preburnettiene $\mathrm{B}$, a recently characterized metabolite from Aspergillus burnetti. ${ }^{38}$

\section{Results}

\section{Design and construction of a recombinase-mediated integration system in} Aspergillus nidulans

We designed an RMCE strategy involving lox sites that contain both the heterospecific mutation of lox $2272^{39}$ and either of the heteromeric (LE/RE) mutations of lox71 and lox66, ${ }^{40}$ creating the sites lox2272-71 and lox2272-66 (Figure 1, Table S1). In principle, lox2272-71 and lox2272-66 recombination is irreversible as it results in the double LE/RE mutant site lox2272-72 (Table S1). Additionally, the sites lox2272/-72/-66/-71 are incompatible with loxP 
bioRxiv preprint doi: https:/doi org/10.1101/2021.08.20.457072; this version posted December $19,2021$. The copyright holder for this preprint (which was not certified by peer review) is the author/funder, who has granted bioRxiv a license to display the preprint in perpetuity. It is made available under aCC-BY 4.0 International license.

for recombination. Therefore, by flanking the donor cassette and landing pad by loxP and lox2272-66 or lox2272-71, RMCE integration can be achieved (Figure 1).

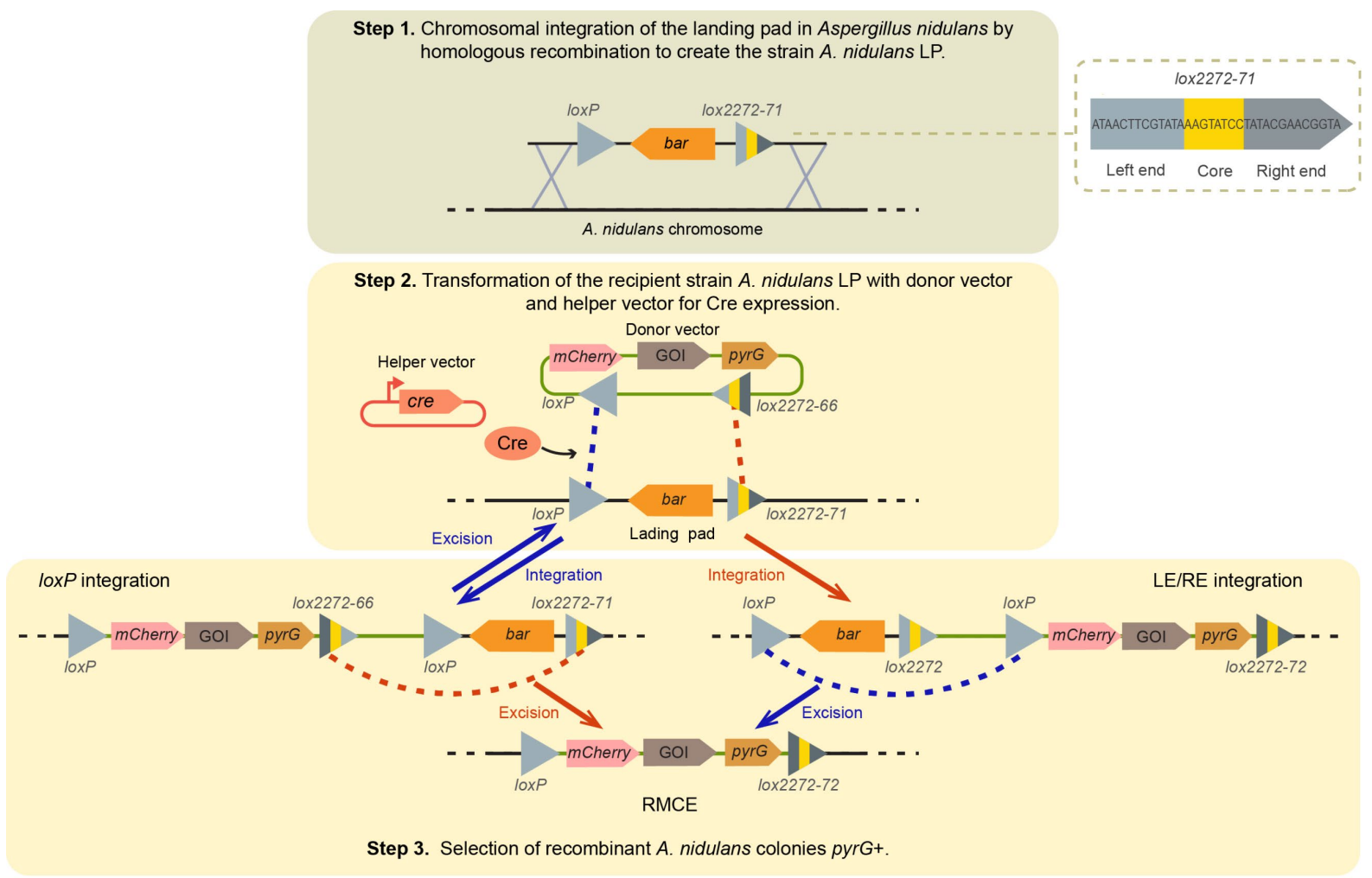

Figure 1 Overview of the strategy for Cre/lox-mediated chromosomal integration (not to scale). Step 1) The short landing pad (LP) containing the bar marker gene flanked by loxP and lox2272-71 is integrated into the destination locus of Aspergillus nidulans by homologous recombination, creating the strain $A$. nidulans LP. Step 2) $A$. nidulans LP protoplasts are transformed with the donor vector, which contains loxP and lox2272-66 flanking the marker gene pyrG, a fluorescent reporter and the genes of interest (GOIs), along with a helper vector for transient expression of Cre recombinase. Cre mediates stable integration by LE/RE recombination or by RMCE in two recombination events (integration and excision). Step 3) The recombinant transformant colonies are selected in minimal media for pyrG complementation. As the strain $A$. nidulans $L P$ is reused in further transformation rounds, step 1 is only performed once.

First, we validated the capability of Cre to recombine the sites lox2272-66 and lox227271 by an assay in vitro (Figure S1). Next, to create the recipient fungal strain, we chromosomally integrated the floxed (flanked by lox sites) landing pad (LP) by homologous recombination in the strain $A$. nidulans LO8030.41 The landing pad consisted of the sites loxP and lox2272-71 (Figure 1, Table S1) flanking the marker bar for glufosinate resistance. We selected as a first landing locus the sterigmatocystin biosynthetic gene cluster boundaries $(\Delta s t c)$, as we have previously used this locus for the chromosomal expression of heterologous genes. ${ }^{9}$ After glufosinate selection of the transformant colonies and PCR verification, the strain A. nidulans landing pad 1 (LP1) was isolated for future tests. 
For transient Cre recombinase expression, we created a helper vector unable to replicate in $A$. nidulans. The helper vector encodes a cassette for constitutive expression of Cre under the promoter $g p d A\left(P_{g p d A}\right)$ and the terminator trpC (Figure 1$)$. As the A. nidulans LP1 recipient strain carries $\triangle n k u A$, which minimizes random integration events, the helper vector presumably would be lost during fungal growth. ${ }^{17}$

To test the feasibility of recombinase-mediated integration at LP1, we built vectors containing donor cassettes with the fluorescent reporter $m$ Cherry and the pyrG marker flanked by IoxP and lox2272-66 (Figure 2A, Figure S2A). Donor_vector-1 is a $6.6 \mathrm{~kb}$ small vector containing a $3.6 \mathrm{~kb}$ floxed donor cassette. Donor_vector-2 is $12.2 \mathrm{~kb}$ shuttle vector that supports yeast transformation-associated recombination cloning and contains a $6.6 \mathrm{~kb}$ floxed donor cassette that incorporates four cloning sites for the expression of biosynthetic genes under alcohol-inducible promoters ${ }^{11}$ or the cloning BGC genes under native promoters (Figure 2a, Figure S2A). To evaluate the integration of a larger donor cassette, we built donor_vector2-bue containing a $21.4 \mathrm{~kb}$ floxed cassette that includes part of the bue BGC from A. burnettii as proof-of-concept (Figure 2a, Figure S2A). The bue BGC has recently been characterized by heterologous episomal expression in $A$. nidulans to produce burnettiene $A .{ }^{38}$ As the complete BGC led to production of multiple metabolite derivatives along with the final product, we decided to evaluate instead the integration of the partial BGC containing bueA/B/C/D/E/R (without bueF) whose expression results in the production of a single peak corresponding to the penultimate pathway product, preburnettiene $B(1) .{ }^{38}$ As the bue cluster is active in the native host ${ }^{42}$ and episomal expression of bue genes under native promoters in $A$. nidulans leads to compound production, ${ }^{38}$ we hypothesized that if the partial bue BGC was chromosomally integrated into $A$. nidulans we would observe the production of 1 (Figure S2B).

\section{Cre/lox-mediated integration of diverse donor cassettes}

To evaluate the efficiency of the recombination system, we transformed protoplasts of A. nidulans LP1 by a small-scale PEG mediated transformation in $2 \mathrm{~mL}$ microtubes (Figure 2A). We tested different amounts and ratios for each donor vector and the helper vector, and we also transformed $A$. nidulans with the donor vector alone as a control. We consistently obtained viable colonies in the strains transformed with the donor vector along with the helper vector for cre expression (3-13 colonies per transformation event) (Table 1). The variation in the number of transformant colonies was independent of the size of the donor fragment, which is expected for recombinase-mediated integration (Table 1). ${ }^{27}$

In the control strains without cre helper vector we mostly observed only small "abortive" colonies that did not support further growth. Abortive colonies arising from residual nonintegrated vector encoding pyrG have been previously reported in $A$. nidulans $\Delta n k u A$ strains. ${ }^{17}$ 
Interestingly, we observed a higher number of abortive colonies when transforming the smaller donor_vector-1 compared to the larger donor_vector-2 and donor_vector-2-bue. Additionally, we did not observe viable colonies in the controls when transforming the larger donor vectors without the cre helper vector (Table 1).

To evaluate the mechanism of integration in the transformant $p y r G^{+}$colonies, we analyzed the landing locus by PCR amplification of the recombination junction regions with sets of primers for the original landing pad or the recombination product (Figure 2B and C, Figure S3). The frequency of successful recombinase-mediated integration ranged from 29$100 \%$ across experiments (Table 1). We observed that lower recombination efficiency was found in transformations carried out with $<0.9 \mathrm{pmol}$ of helper vector. At higher amounts of helper vector, Cre-mediated recombination efficiency for donor_vector-1 ranged from 60-83\% (Table 1). Importantly, the recombination efficiency of the larger vectors donor_vector-2, and 2-bue ranged between $90-100 \%$ at the highest helper vector concentrations (Table 1, Figures S3D and E). The lower frequency of false positives seems to indicate that larger donor vectors are less prone to random integration. We also observed variability between the type of recombination output (RMCE, LE/RE integration only or loxP integration only) with varying donor vector and helper vector amounts (Figure 2E, Figure S3B). At this stage, we also confirmed by sequencing of PCR products that the recombination product between lox227266 and lox2272-71 in vivo is the double LE/RE mutant site lox2272-72 (Figure 2D).

To evaluate the recombination system at a different loci, we created an $A$. nidulans strain harboring a landing pad in the locus IS1 (LP2), which has been used for heterologous BGC expression (Figure 2, Figure S4A). ${ }^{18}$ When evaluating integration of donor_vector-2-bue at LP2 with sufficient cre helper vector we obtained $100 \%$ efficiency of recombinase-mediated integration in both experiments mostly by RMCE (Table 1, Figure S4B-D).

To investigate if the helper vector is lost in the absence of selection pressure, we analyzed the presence of the cre gene by PCR, and obtained positive results in several colonies across experiments (Figure S5). These results could imply that traces of the residual vector might be retained at later growth stages or that random integration of the cre helper vector occurs in A. nidulans.

Table 1 Cre/lox-mediated integration experiments in Aspergillus nidulans using nonreplicative donor vectors, with landing pad (LP) parent strain and negative controls (C) indicated. 


\begin{tabular}{|c|c|c|c|c|c|}
\hline Donor vector & LP & $\begin{array}{c}\text { Transformation } \\
\text { event }\end{array}$ & $\begin{array}{l}\text { Donor } \\
\left(\mathrm{pmol}^{\mathrm{a}}\right)\end{array}$ & $\begin{array}{l}\text { Helper } \\
\left(\text { pmola }^{a}\right)\end{array}$ & $\begin{array}{c}\text { Colonies with } \\
\text { Cre/lox-mediated } \\
\text { integration from } \\
\text { total }^{\mathrm{b}}\end{array}$ \\
\hline \multirow{5}{*}{$\begin{array}{l}\text { Donor_vector-1 } \\
\text { (3.8 kb donor } \\
\text { cassette / } 6.6 \mathrm{~kb} \\
\text { vector) }\end{array}$} & \multirow[t]{5}{*}{ LP1 } & 1 & 0.63 & 2.41 & $3 / 5(60 \%)$ \\
\hline & & 2 & 1.10 & 1.67 & $5 / 6(83 \%)$ \\
\hline & & 3 & 0.37 & 0.83 & $2 / 7(29 \%)$ \\
\hline & & C1 & 1.10 & - & $0 / 1(0 \%)$ \\
\hline & & $\mathrm{C} 2$ & 0.37 & - & $0 / 1(0 \%)$ \\
\hline \multirow{2}{*}{$\begin{array}{c}\text { Donor_vector-2 } \\
(6.3 \mathrm{~kb} \text { donor } \\
\text { cassette / } 12.2 \mathrm{~kb} \\
\text { vector })\end{array}$} & \multirow[t]{2}{*}{ LP1 } & 1 & 0.94 & 1.67 & $12 / 13(92 \%)$ \\
\hline & & C1 & 0.94 & - & No colonies ${ }^{\mathrm{c}}$ \\
\hline \multirow{9}{*}{$\begin{array}{c}\text { Donor_vector-2-bue } \\
\text { (21.4 kb donor } \\
\text { cassette / } 27.3 \mathrm{~kb} \\
\text { vector })\end{array}$} & \multirow[t]{6}{*}{ LP1 } & 1 & 0.53 & 1.67 & $9 / 9(100 \%)$ \\
\hline & & 2 & 0.64 & 1.67 & $5 / 5(100 \%)$ \\
\hline & & 3 & 0.64 & 0.33 & $1 / 3(33 \%)$ \\
\hline & & C1 & 0.53 & - & No colonies $^{c}$ \\
\hline & & $\mathrm{C} 2$ & 0.64 & - & No colonies ${ }^{c}$ \\
\hline & & C3 & 0.64 & - & No colonies ${ }^{c}$ \\
\hline & \multirow[t]{3}{*}{ LP2 } & 1 & 0.47 & 2.25 & $4 / 4(100 \%)$ \\
\hline & & 2 & 0.47 & 2.25 & $3 / 3(100 \%)$ \\
\hline & & C1 & 0.47 & - & No colonies ${ }^{c}$ \\
\hline
\end{tabular}

${ }^{a}$ Estimated based on vector concentration obtained by Nanodrop after standard miniprep, which might overestimate DNA concentration.

${ }^{\mathrm{b}}$ Per reaction $3-6 \times 10^{6} \mathrm{~A}$. nidulans protoplasts were used.

${ }^{\mathrm{c}}$ No viable colonies. Abortive colonies were inoculated in fresh media, but no growth was observed. 


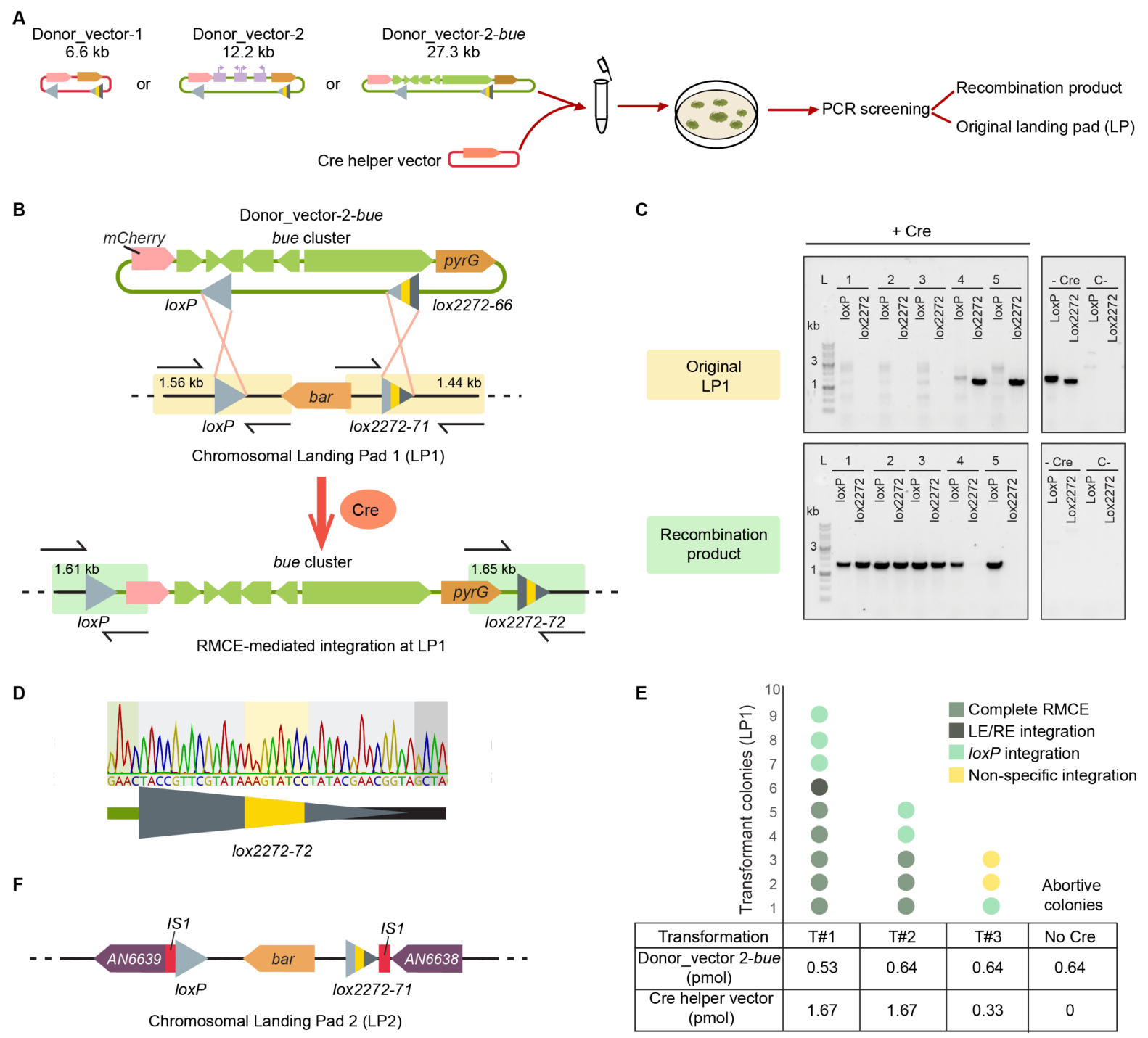

Figure 2. Efficient targeted chromosomal integration of the bue biosynthetic gene cluster in Aspergillus nidulans A. Experimental setup for the evaluation of donor vectors. B. Schematic the strategy for bue cluster genes integration at landing pad 1 (LP1). PCR amplicons are represented by colored with sizes indicated. C. Representative PCR results of transformant colonies for donor_vector-2-bue integration. Colonies are indicated as numbers, and the complete gel is found in Figure S3. D. Confirmation of the expected recombination event by Sanger sequencing. E. Amount of transformant colonies obtained in different transformation experiments with donor_vector-2-bue, with the integration mechanism indicated as a color code. F. Schematic of landing pad 2 (LP2) in A. nidulans IS1 locus.

\section{Phenotypic stability of recombinant Aspergillus nidulans strains}

To evaluate the recombinant strains we first analyzed the expression of a $P_{g p d A^{-}}$ $m$ Cherry fluorescent reporter encoded in the cassettes integrated at LP1 or LP2. We consistently observed fluorescence in the mycelia of the recombinant strains compared to the negative control (Figure 3A, Figure S6). To benchmark chromosomal expression, we compared the recombinant strains to strains expressing mCherry from an episomal AMA1pyrG vector. We observed that AMA1-based expression was stronger than the chromosomally 
integrated counterparts when the mycelia was grown under selective conditions, but AMA1based expression was mostly lost in mycelia grown under non-selective conditions (Figure 3A, Figure S6).

Inspired by comparative studies of chromosomal and episomal expression in yeast by Jensen et $a l,{ }^{43}$ we analyzed the spores of recombinant colonies by flow cytometry to test phenotypic stability. We observed a unimodal distribution of fluorescence in the strains with $m$ Cherry integrated at LP1 or LP2, distinguishable from the negative control (Figure 3C, Figure S7). The strains with AMA1-based episomal expression of $m$ Cherry presented a much wider multimodal distribution, indicative of the heterogenous expression of AMA1-encoded genes on spores even under selective conditions (Figure 3C, Figure S7). ${ }^{13}$ While these results indicated that chromosomal expression at LP1 or LP2 results in a more homogeneous cell population, expression in the recombinant strains was at least one order of magnitude lower than the best performing spores from strains with AMA1-episomal expression (Figure 3C, Figure S7).

To further evaluate the expression pattern of mCherry in spores during fungal growth in solid media, we analyzed the recombinant strains at LP2 by fluorescent photography (Figure 3B, Figure S8). We observed that the recombinant strains for chromosomal expression showed uniform fluorescence in selective and non-selective conditions, distinguishable from the negative control. In contrast, the strains with AMA1-encoded mCherry presented a patchy expression pattern across the fungal colony both under selective and non-selective conditions (Figure 3B, Figure S8). Subsequent spore dilutions showed AMA1-based mCherry expression was lost in most colonies under non-selective conditions, but interestingly some colonies in non-selective media retained fluorescence (Figure S8B). Overall, our analysis of the fluorescent reporter at LP1 or LP2 supported phenotypic stability, which is expected of chromosomally integrated genes. 
A

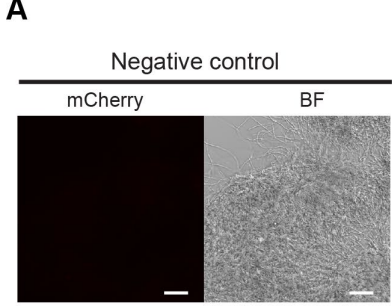

B

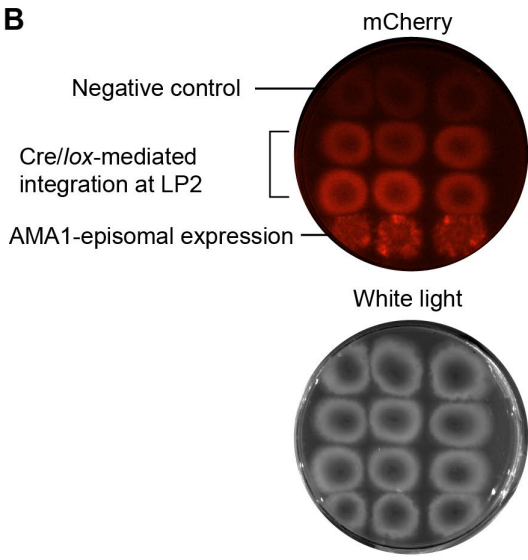

$P_{\text {gpdA }}$ - $m$ Cherry chromosomally integrated (LP1)

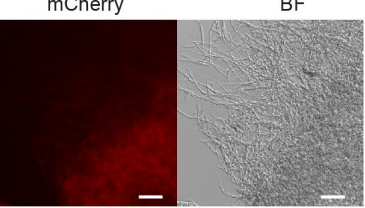

C

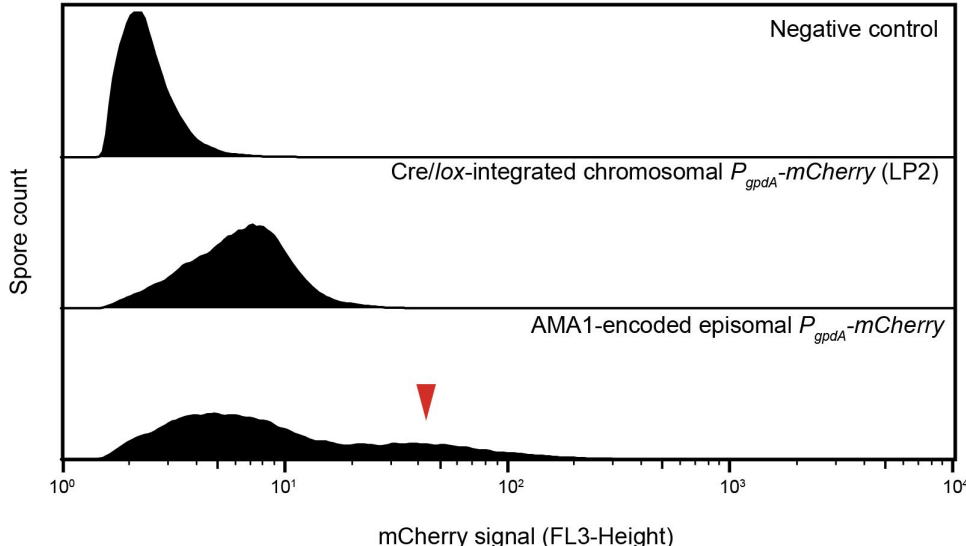

$P_{\text {gpda }}-$-mCherry

encoded

non-selective conditions
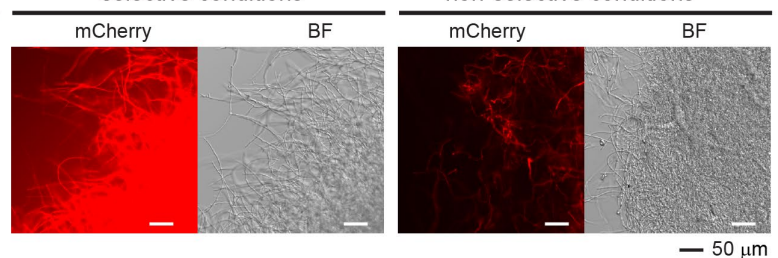

$-50 \mu \mathrm{m}$

Cherry signal (FL3-Height)

Figure 3. Expression of mCherry integrated at the chromosomal landing pads (LPs) compared to AMA1-based episomal expression. A. Strains with mCherry chromosomally integrated at LP1 show consistent but lower fluorescence than the AMA1-based episomal counterpart under selective conditions. Selection pressure is needed to support high AMA1-based episomal expression. Samples with similar mycelial growth were observed under mCherry filter and brightfield (BF) by fluorescence microscopy. Biological replicates are found in Figure S6. B. The expression in recombinant $A$. nidulans colonies at LP2 is more homogeneous than the strains encoding mCherry episomally, which show a patchy expression pattern even under selective conditions. Extended information in Figure S8. C. Analysis of spores by flow cytometry shows more compact and homogeneous fluorescence in samples with chromosomal integration of $m$ Cherry at LP2 compared to AMA1-based episomal expression. A proportion of the spores expressing mCherry episomally from AMA1 vectors can reach fluorescence levels one order of magnitude higher than spores with chromosomal expression at LP2 (red arrow). Extended information in Figure S7.

\section{Expression of the polyketide preburnettiene B from chromosomally integrated}

\section{genes}

To assess the production of the heterologous polyketide 1 , we cultivated recombinant strains with bueA/B/C/D/E/R chromosomally integrated at LP1 or LP2. Surprisingly, we did not observe detectable production of 1 when the genes were chromosomally integrated, while we consistently observed the production of 1 in the strains with bue genes episomally encoded on AMA1 vector (Figure S9). For troubleshooting, we verified the correct integration of bue genes in recombinant strains by whole genome sequencing (Figure S10). Thus, and given 
that mCherry was still expressed (Figure S9), the lack of compound production could be due to the bue genes being silent when integrated chromosomally.

We previously demonstrated that overexpression of the bue cluster-specific transcription factor (TF) bueR increased the production of burnettienes in an episomal context. ${ }^{38}$ Therefore, we hypothesized that overexpression of the TF bueR would activate the expression of the chromosomal bue genes in the recombinant $A$. nidulans strains (Figure 4A). Effectively, we observed the production of 1 in strains harboring bue genes integrated by RMCE at either LP1 or LP2 when further transformed with an AMA1-based vector encoding $P_{g p d A}-b u e R$ and the transporter gene bueG ${ }^{38}$ (Figure $4 \mathrm{~B}$ and $\mathrm{C}$ ). This result validates the presence of a functional and complete integrated copy of bueA/B/C/D/E/R. When comparing strains with bue genes integrated at LP1 and LP2, we observed similar production levels between them (Figure 4C).

One of the benefits of chromosomal expression is that cultures can be grown under nonselective conditions without compromising productivity. For simplicity, in previous small-scale culturing experiments we cultivated the strains on liquid minimal media with no uracil and uridine, the selective condition of the pyrG marker. To evaluate expression under nonselective conditions, we cultivated strains containing the bue genes at LP2 and TF overexpression in media supplemented with uracil and uridine. It should be noted that the AMA1-based vector for TF expression is under the selection of pyridoxal auxotrophies with the pyroA marker and the medium used lack pyridoxine. As expected, pyrG selection pressure was not needed for compound production (Figure 4B and C). Interestingly, we observed a 3fold higher production in the strains grown in non-selective conditions (Figure 4C). Positional effects arising from insufficient expression of the pyrG marker integrated in some loci have been observed in $A$. nidulans before, thus supplementation with uracil and uridine could improve the strain fitness. ${ }^{44,45}$ To confirm that the TF BueR alone was responsible for the activation of bue genes, we evaluated strains lacking the transporter bueG. Interestingly, we observed even higher production of 1 in the strains with bueR overexpression alone compared to the strains additionally expressing bueG (Figure S11).

To sum up, overexpression of the cluster-specific TF bueR activated the expression of bue genes at two different chromosomal contexts. These results demonstrate that recombinase-mediated integration is a feasible strategy to build strains for heterologous compound production, but that alternative strategies might be needed for BGC activation if the gene cluster remains silent. Overall, chromosomal expression permitted cultivating in nonselective media without compromising yields. 
A
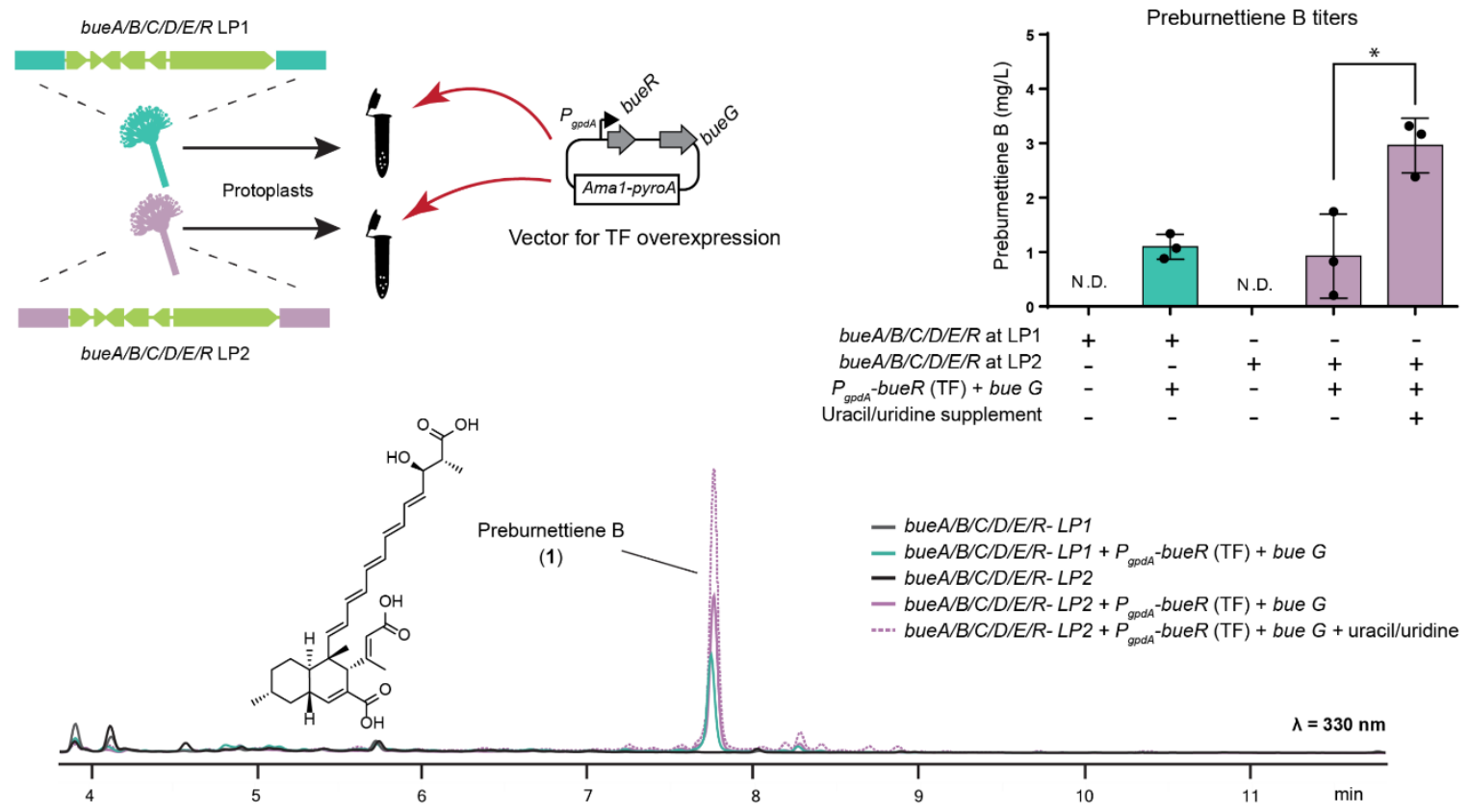

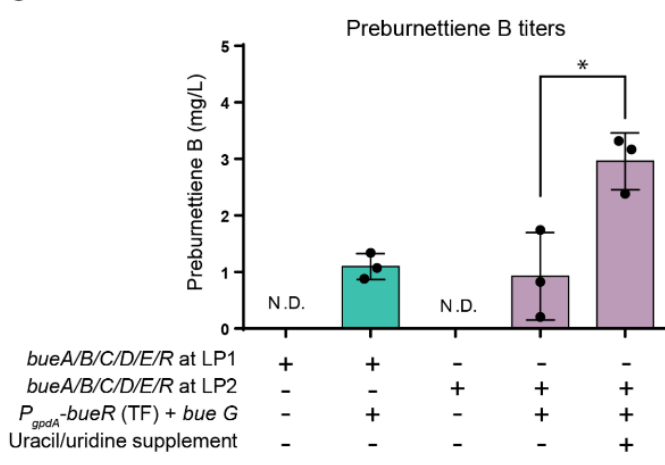

Figure 4. Activation of chromosomally integrated bue cluster genes by transcription factor (TF) overexpression. A. Protoplasts were prepared from strains with RMCE integrated bue genes at LP1 or LP2, and further transformed with a vector for bueR overexpression, that additionally encodes the bue $G$ transporter. B. Chromatogram traces at $\lambda=330 \mathrm{~nm}$ show the activation of the production of preburnettiene B (1) in strains with TF overexpression. C. Comparable compound titers are observed between the strains with bue genes integrated at LP1 or LP2 with TF overexpression on minimal media. Strains grown on media supplemented with uracil and uridine (non-selective condition) presented higher titers than the same strain grown on minimal media (selective condition). Values are the mean of three biological replicates, specific values are indicated as black dots, and error bars represent SD. Twosided Welch's T-test $p$-value $=0.024$.

\section{Discussion}

In this work we established a system for Cre/lox-mediated chromosomal integration of large heterologous BGCs in $A$. nidulans. Site-specific recombinase mediated integration represents a relevant expansion to the synthetic biology toolbox for filamentous fungi, where recombinases had only been used for gene deletion or inversion. ${ }^{37}$ The vector set developed in this work can be adapted for integration at different chromosomal landing loci by replacing the homology arms in the landing pad. Additionally, the vectors are built with promoters and markers portable to other fungi ( $P_{g p d A}, T_{t r p c}$, bar, pyrG), and therefore could be used to integrate large heterologous DNA in diverse fungal cell factories. ${ }^{22}$

We demonstrated targeted one-step integration of $21 \mathrm{~kb}$ DNA regions by RMCE in $A$. nidulans, and this system could be used for integrating larger constructs in future works. We obtained high transformation efficiency (up to 100\%) using a small-scale transformation protocol with optimized amounts of helper vector. We additionally observed that the false positive rate and the presence of abortive colonies diminished when transforming large donor 
vectors ( $\geq 12 \mathrm{~kb}$ ). We also demonstrated that the resulting strains with chromosomally encoded genes presented a more uniform fluorescence phenotype in spores and mycelia compared to AMA1-encoded genes, evidencing genetic and phenotypic stability.

The developed system complements current methods for targeted gene integration in filamentous fungi which rely on DNA repair pathways, such as HR or CRISPR-based knock in (KI). CRISPR-KI has been mostly used to integrate fragments that span few kilobases, and although recently it has been used for the integration of larger BGC fragments in Aspergilli, the efficiency of targeted integration is not reported. ${ }^{20,21}$ In contrast, recombinase-mediated integration does not generate dsDNA breaks and is orthogonal to the host machinery. This makes recombinase-based systems a convenient and reliable alternative for the single-step integration of large DNA constructs, where the efficiency of HR declines. However, recombinase-mediated integration also faces the same constraints as HR for heterologous expression featuring a single gene copy in a chromosomal context. When integrating the heterologous genes bueA/B/C/D/E/R under their native promoter at LP1 or LP2 initially we did not observe compound production. Nevertheless, when overexpressing the cluster specific TF bueR we restored compound production from the bue genes at LP1 and LP2. Strategies for BGC activation, such as TF overexpression, promoter replacement or CRISPRa could be used to activate chromosomally integrated genes that remain silent. ${ }^{9}$ Presumably, integration in a better locus for expression could also result in improved performance. ${ }^{22}$

By benchmarking chromosomal expression to AMA1 based expression, this work also represents an unprecedented characterization of AMA1 episomal expression under the marker pyrG in A. nidulans. Our original analysis of AMA1-pyrG phenotypic stability by flow cytometry adds a quantitative estimation of the phenotypic heterogeneity of AMA1-based expression in spores from in A. nidulans grown under selective conditions in solid media. Even though our results indicate that the phenotypic stability of AMA1-encoded genes in spores is limited, we observed that during mycelial growth in liquid culture there is a more prevalent phenotype for strong expression in both fluorescence and compound production.

Lastly, the Cre/lox-mediated integration platform can be expanded in future works. Cre/lox-mediated integration could be particularly relevant for integrating large genomic regions containing BGCs cloned by genome capture. ${ }^{12}$ Donor_vector-2 currently supports the assembly of four genes under strong promoters by homology-based cloning, but future donor vectors could be adapted for Type-lls modular cloning to facilitate the assembly of multigene BGCs where each gene needs promoter replacement. ${ }^{46,47}$ The current system could also be upgraded for simultaneous integration in different loci by using multiple landing pads with different heterospecific recombination sites. To minimize the risk of integration of the helper vector, strategies such as self-excising Cre expression cassettes could also be evaluated. ${ }^{28}$ 
Additionally, other recombinase systems could be explored for integration in filamentous fungi. 48

To sum up, Cre/lox-mediated integration of BGCs has the potential to speed up the process of constructing strains to produce heterologous metabolites in $A$. nidulans. The capability to uptake and maintain complex exogenous DNA is a key requirement for a good chassis organism for bioproduction. ${ }^{49}$ Thus, this system can be used to upgrade other filamentous fungi chassis. Importantly, this system could be adapted to integrate other large DNA constructs in commonly used industrial Aspergilli for other biotechnological applications. ${ }^{2}$

\section{Methods}

\section{Vector Construction}

All vectors are listed in Table S2 and the oligonucleotides used are listed in Table S4 with their destination vector indicated. Vectors were constructed by isothermal assembly or digestion with restriction site enzymes and ligation. Cloning strategies for each vector are detailed in Supporting Methods. Cre was amplified from pBF3038. ${ }^{50}$ Relevant vectors will be made available via Addgene (Table S2).

\section{Aspergillus nidulans strains construction and transformation}

Genotype of parental strains are listed in Table S3. A. nidulans parental strains with LP1 or LP2 were created by polyethylene glycol (PEG)-calcium-based transformation ${ }^{51}$ with Notl linearized vectors pGemLP1-loxP-bar-Lox2272-71 or pGemLP2-loxP-bar-Lox2272-71 containing $1 \mathrm{~kb}$ homology regions for homologous recombination in A. nidulans L08030. Colonies were selected for resistance to glufosinate extracted from Basta as previously ${ }^{9}$, an the event was confirmed by diagnostic PCR.

Protoplasts of $A$. nidulans LO8030 LP1 or LP2 were prepared from germlings, ${ }^{51}$ mixed with a quarter volume PEG $60 \%$ to a final concentration of $10^{8}$ protoplasts per $\mathrm{mL}$ and frozen at $-80^{\circ} \mathrm{C}$ for later use. For $A$. nidulans transformation, , $60 \mu \mathrm{L}$ of thawed protoplast solution in a $2 \mathrm{~mL}$ microcentrifuge tube was incubated with $40 \mu \mathrm{L}$ of STC buffer (1.2 M sorbitol, $10 \mathrm{mM}$ $\mathrm{CaCl}_{2}, 10 \mathrm{mM}$ Tris- $\mathrm{HCl}, \mathrm{pH}$ 7.5) and the vector amounts indicated in Table 1. After 20 min of incubation on ice, $400 \mu \mathrm{L}$ of the calcium PEG $60 \%$ mix was added and mixed gently by inversion, followed by a 20 min incubation at room temperature. After adding $1 \mathrm{~mL}$ of STC buffer the mix was spread on two plates of stabilized minimal media (SMM) supplemented with pyridoxine $(\mathrm{p}+)$ and riboflavin $(\mathrm{r}+)$ but lacking uracil or uridine $(\mathrm{u}-)$ for auxotrophic selection $(u-p+r+)$, that were then incubated for three days at $37^{\circ} \mathrm{C}$ to generate transformant colonies. 
Relevant strains of $A$. nidulans $L 08030-L P 1-b u e A / B / C / D / E / R$ and $A$. nidulans $L 08030$ $L P 2-b u e A / B / C / D / E / R$ were analyzed by whole genome sequencing with the DNBSEQ-2000 PE150 platform at BGI Tech Solutions co. (BGI, Hong Kong), resulting in 2 Gb of raw genome data (150 bp, paired-end). The reads were mapped to chromosome sequences containing the expected recombination product using Geneious 11.03 .

For the activation of the chromosomally integrated bue genes by TF overexpression, protoplasts were prepared from the strains $A$. nidulans $L 08030-L P 1-b u e A / B / C / D / E / R$ and $A$. nidulans $L 08030-L P 2-b u e A / B / C / D / E / R$. Protoplasts were transformed with the vector pYFACbueG-PgpdA-bueR ${ }^{38}$ or pYFAC- PgpdA-bueR and selected on SMM u-r+p-.

\section{Diagnostic PCRs}

Genomic DNA (gDNA) was extracted from mycelial mass after overnight growth in liquid glucose minimal media (GMM) $u-p+r+$ at $37^{\circ} \mathrm{C}$. The PCR amplification was conducted by 3 min at $94{ }^{\circ} \mathrm{C}$ initial denaturing followed by 27 cycles of $30 \mathrm{~s}$ at $94{ }^{\circ} \mathrm{C}, 30 \mathrm{~s}$ at $56.5^{\circ} \mathrm{C}, 60 \mathrm{~s}$ at $72{ }^{\circ} \mathrm{C}$, in a thermocycler with Taq polymerase, using $\sim 30 \mathrm{ng}$ of $A$. nidulans gDNA in a $10 \mu \mathrm{l}$ reaction volume. The PCR products were run in a $0.8 \%$ agarose gel with $2 \mu$ of the AccuRuler $1 \mathrm{~kb}$ DNA RTU Ladder (Maestrogen). All agarose gel images were acquired with a Vilber EBox VX-2 gel imager with software V15.10. Representative PCRs products were amplified with Pfu polymerase for Sanger sequencing.

\section{Fluorescence microscopy}

Spores from three or more individual colonies were picked and grown overnight at $37^{\circ}$ $C$ in small petri dishes containing liquid GMM $u-p+r+$ or $u+p+r+$. Fluorescence images were captured at 20x on the epifluorescence inverted microscope Eclipse Ti2 (Nikon), using numerical aperture (NA) 0.75 and Plan Apo $\lambda$ 20x objective lens (Nikon) and a Camera DSQi2 (Nikon) controlled by NIS Elements Advanced Research (Nikon). Fluorescent microscopy was carried out under a mCherry filter set (562/40 nm excitation, $593 \mathrm{~nm}$ dichroic beamsplitter, and $641 / 75 \mathrm{~nm}$ emission), using a $400 \mathrm{~ms}$ exposure and 1.8x analog gain unless specified otherwise. Images were recorded using NIS-Elements Advanced Research AR V4.60 software package (Nikon).

\section{Flow cytometry}

Spores were collected in water with a sterile loop from colonies grown for 3 days in solid GMM $u-p+r+r$. The spore suspension was filtered through a syringe containing sterile cotton to remove residual mycelia and diluted to a concentration of $\sim 1.10^{6} \mathrm{spores} / \mathrm{mL}$. Data acquisition was performed immediately after spore suspension using a FACSCalibur (BD 
Biosciences) flow cytometer operated with filtered water as sheath fluid. mCherry signal was observed with a $488 \mathrm{~nm}$ excitation laser and the filter FL3 ( $\geq 670 \mathrm{~nm}$ ) and 35,000 events were recorded per sample or events within 4 minutes of run time as collected for the filtered water control. The data was processed using FlowJo V10 software (TreeStar). The output was gated according to forward scatter (FCS) to limit to the size range of spores, an example of the gating strategy is indicated on the Figure S7.

\section{Fluorescent photography}

Plates were analyzed in ChemiDoc MP Imaging System (Bio-Rad) and images recorded with ImageLab software V6.1.0 (Bio-Rad). MCherry images were obtained using the excitation source Green Epi illumination and the emission filter 605/50 nm with exposure time of 0.01 seconds. Bright field was captured with white illumination and a standard emission filter in automatic exposure.

\section{Metabolic profile analysis by LC-DAD-MS}

For each transformant strain, spores from individual colonies were re-streaked individually in a solidified GMM (u-p+r+or $u-p-r+$ ) plate and cultivated for three days at $37{ }^{\circ} \mathrm{C}$. Spores were harvested from plates in $1 \mathrm{~mL}$ of $0.1 \%$ Tween 80 (Sigma, MO, USA) and approximately $10^{8}$ spores were inoculated into $250-\mathrm{mL}$ flasks containing $50 \mathrm{~mL}$ liquid GMM $\left(\mathrm{u}-\mathrm{p}+\mathrm{r}+, \mathrm{u}-\mathrm{p}-\mathrm{r}+\right.$ or $\mathrm{u}+\mathrm{p}-\mathrm{r}+$ ) medium. Additionally, ampicillin was added to $50 \mu \mathrm{g} \mathrm{mL}^{-1}$. Cultures were incubated for 4 days with shaking set to $200 \mathrm{rpm}$ and $26{ }^{\circ} \mathrm{C}$. At the end of the culture, 20 $\mathrm{mL}$ of media was collected in $50-\mathrm{mL}$ falcon tubes by filtration with Miracloth (Milipore, MA, USA). The metabolites were extracted from the liquid culture with $20 \mathrm{~mL}$ of an organic solvent mixture containing ethyl acetate, methanol, and acetic acid (89.5: $10: 0.5$ ratio). The crude extracts were dried down in vacuo and re-dissolved in $0.3 \mathrm{~mL}$ of methanol for LC-DAD-MS analysis.

The analyses of the metabolite profiles were performed on an Agilent 1260 liquid chromatography (LC) system coupled to a diode array detector (DAD) and an Agilent 6130 Quadrupole mass spectrometer (MS) with an electrospray ionization (ESI) source. In all cases $3 \mu \mathrm{L}$ of the methanol dissolved crude extract was injected. Chromatographic separation was performed at $40{ }^{\circ} \mathrm{C}$ using a Kinetex C18 column (2.6 $\mu \mathrm{m}, 2.1 \mathrm{~mm}$ i.d. x $100 \mathrm{~mm}$; Phenomenex). Chromatographic separation was achieved with a linear gradient of 5-95\% acetonitrile-water (containing $0.1 \% \mathrm{v} / \mathrm{v}$ formic acid) in 10 minutes followed by $95 \%$ acetonitrile for 3 minutes, with a flow rate of $0.70 \mathrm{~mL} \mathrm{~min}{ }^{-1}$. The MS data were collected in the $\mathrm{m} / \mathrm{z}$ range $100-1000$ in negative ion mode and UV observed at DAD $\lambda=330 \mathrm{~nm}$. Peak areas were determined by integration using Masshunter Workstation Qualitative Analysis B.07.00 (Agilent). To quantify 
preburnettiene B (1) samples were compared to a calibration curve prepared with a pure standard (Microbial Screening Technologies) and extrapolated to titers of burnettiene per liter (Figure S12). Statistical analysis was performed with GraphPad Prism 8.3.0 by a Two-sided Welch's T-test using biological replicates.

\section{Abbreviations}

BGCs, biosynthetic gene clusters; dsDNA, double stranded DNA; CRISPR, clustered regularly interspaced short palindromic repeat; $\mathrm{HR}$, homologous recombination; Kb, kilobase; $\mathrm{KI}$, knock in; LE, left end ; LE/RE, Left/Right ends recombination; LP, landing pad; PKS, polyketide synthase; Re, right end; RMCE, Recombinase Mediated Cassette Exchange; SM, secondary metabolite; TF, transcription factor.

\section{Supporting Information}

Supporting Methods: cloning strategy and in vitro recombination; Figures S1-12: Biological replicates of fluorescence microscopy and flow cytometry, fluorescent photography, donor vector schematics, sequencing results, LC/MS analysis, PCR gels, in vitro recombination, calibration curve; Tables S1-4: lox sites used, primers, vectors and strains used in this work.

\section{Acknowledgments}

This project is supported by the Australian Research Council (FT160100233 and DP210102180). I.R. was recipient of an UWA PhD Scholarship. We thank Microbial Screening Technologies (MST) for the strain Aspergillus burnettii MST-FP2249 and the preburnettiene B standard. A. nidulans LO8030 is a gift from Berl Oakley. We thank Hamideh Rezaee for her help building donor_vector-2, and Julia Grassl (UWA) for helping us to set up the flow cytometer.

\section{Conflicts of interest}

There are no conflicts to declare.

\section{Author contribution}

I.R and Y.H.C conceived the project and wrote the manuscript. I.R. designed and performed the experiments and the data analysis. 


\section{References}

(1) Wakai, S., Arazoe, T., Ogino, C., and Kondo, A. (2017) Future insights in fungal metabolic engineering. Bioresour. Technol. 245, 1314-1326.

(2) Meyer, V., Basenko, E. Y., Benz, J. P., Braus, G. H., Caddick, M. X., Csukai, M., de Vries, R. P., Endy, D., Frisvad, J. C., Gunde-Cimerman, N., Haarmann, T., Hadar, Y., Hansen, K., Johnson, R. I., Keller, N. P., Kraševec, N., Mortensen, U. H., Perez, R., Ram, A. F. J., Record, E., Ross, P., Shapaval, V., Steiniger, C., van den Brink, H., van Munster, J., Yarden, O., and Wösten, H. A. B. (2020) Growing a circular economy with fungal biotechnology: a white paper. Fungal Biol. Biotechnol. 7, 5.

(3) Keller, N. P. (2019) Fungal secondary metabolism: regulation, function and drug discovery. Nat. Rev. Microbiol. 17, 167-180.

(4) Robey, M. T., Caesar, L. K., Drott, M. T., Keller, N. P., and Kelleher, N. L. (2021) An interpreted atlas of biosynthetic gene clusters from 1,000 fungal genomes. Proc. Natl. Acad. Sci. 118, e2020230118.

(5) Anyaogu, D. C., and Mortensen, U. H. (2015) Heterologous production of fungal secondary metabolites in Aspergilli. Front. Microbiol. 6, 77.

(6) He, Y., Wang, B., Chen, W., Cox, R. J., He, J., and Chen, F. (2018) Recent advances in reconstructing microbial secondary metabolites biosynthesis in Aspergillus spp. Biotechnol. Adv. 36, 739-783.

(7) Caesar, L. K., Kelleher, N. L., and Keller, N. P. (2020) In the fungus where it happens: history and future propelling Aspergillus nidulans as the archetype of natural products research. Fungal Genet. Biol. 144, 103477.

(8) Hu, J., Sarrami, F., Li, H., Zhang, G., Stubbs, K. A., Lacey, E., Stewart, S. G., Karton, A., Piggott, A. M., and Chooi, Y.-H. (2019) Heterologous biosynthesis of elsinochrome A sheds light on the formation of the photosensitive perylenequinone system. Chem. Sci. 10, 14571465.

(9) Roux, I., Woodcraft, C., Hu, J., Wolters, R., Gilchrist, C. L. M., and Chooi, Y.-H. (2020) CRISPR-Mediated Activation of Biosynthetic Gene Clusters for Bioactive Molecule Discovery in Filamentous Fungi. ACS Synth. Biol. 9, 1843-1854.

(10) Liu, L., Tang, M.-C., and Tang, Y. (2019) Fungal Highly Reducing Polyketide Synthases Biosynthesize Salicylaldehydes that are Precursors to Epoxycyclohexenol Natural Products. J. Am. Chem. Soc. 141, 19538-19541. 
(11) Hu, J., Sarrami, F., Li, H., Zhang, G., Stubbs, K. A., Lacey, E., Stewart, S. G., Karton, A., Piggott, A. M. M., and Chooi, Y.-H.(2019) Heterologous biosynthesis of elsinochrome A sheds light on the formation of the photosensitive perylenequinone system. Chem. Sci. 10, 14571465.

(12) Clevenger, K. D., Bok, J. W., Ye, R., Miley, G. P., Verdan, M. H., Velk, T., Chen, C., Yang, K. H., Robey, M. T., Gao, P., Lamprecht, M., Thomas, P. M., Islam, M. N., Palmer, J. M., Wu, C. C., Keller, N. P., and Kelleher, N. L. (2017) A scalable platform to identify fungal secondary metabolites and their gene clusters. Nat. Chem. Biol. 13, 895-901.

(13) Aleksenko, A., and Clutterbuck, A. J. (1997) Autonomous plasmid replication in Aspergillus nidulans : AMA1 and MATE elements. Fungal Genet. Biol. 21, 373-387.

(14) Li, L., Liu, X., Wei, K., Lu, Y., and Jiang, W. (2019) Synthetic biology approaches for chromosomal integration of genes and pathways in industrial microbial systems. Biotechnol. Adv. 37, 730-745.

(15) Ferreira, J. A., Mahboubi, A., Lennartsson, P. R., and Taherzadeh, M. J. (2016) Waste biorefineries using filamentous ascomycetes fungi: Present status and future prospects. Bioresour. Technol. 215, 334-345.

(16) Lubertozzi, D., and Keasling, J. D. (2009) Developing Aspergillus as a host for heterologous expression. Biotechnol. Adv. 27, 53-75.

(17) Nayak, T., Szewczyk, E., Oakley, C. E., Osmani, A., Ukil, L., Murray, S. L., Hynes, M. J., Osmani, S. A., and Oakley, B. R. (2006) A versatile and efficient gene-targeting system for Aspergillus nidulans. Genetics 172, 1557-1566.

(18) Nielsen, M. T., Nielsen, J. B., Anyaogu, D. C., Holm, D. K., Nielsen, K. F., Larsen, T. O., and Mortensen, U. H. (2013) Heterologous Reconstitution of the Intact Geodin Gene Cluster in Aspergillus nidulans through a Simple and Versatile PCR Based Approach. PLoS One 8, e72871.

(19) Yin, W. B., Chooi, Y. H., Smith, A. R., Cacho, R. A., Hu, Y., White, T. C., and Tang, Y. (2013) Discovery of cryptic polyketide metabolites from dermatophytes using heterologous expression in Aspergillus nidulans. ACS Synth. Biol. 2, 629-634.

(20) Jiang, Y., Ozaki, T., Liu, C., Igarashi, Y., Ye, Y., Tang, S., Ye, T., Maruyama, J. I., Minami, A., and Oikawa, H. (2021) Biosynthesis of Cyclochlorotine: Identification of the Genes Involved in Oxidative Transformations and Intramolecular O, N-Transacylation. Org. Lett. 23, 26162620.

(21) Jiang, Y., Ozaki, T., Harada, M., Miyasaka, T., Sato, H., Miyamoto, K., Kanazawa, J., Liu, 
C., Maruyama, J. ichi, Adachi, M., Nakazaki, A., Nishikawa, T., Uchiyama, M., Minami, A., and Oikawa, H. (2020) Biosynthesis of Indole Diterpene Lolitrems: Radical-Induced Cyclization of an Epoxyalcohol Affording a Characteristic Lolitremane Skeleton. Angew. Chemie - Int. Ed. $59,17996-18002$.

(22) Jarczynska, Z. D., Rendsvig, J. K. H., Pagels, N., Viana, V. R., Nødvig, C. S., Kirchner, F. H., Strucko, T., Nielsen, M. L., and Mortensen, U. H. (2021) DIVERSIFY: A Fungal Multispecies Gene Expression Platform. ACS Synth. Biol. 10, 579-588.

(23) Olorunniji, F. J., Rosser, S. J., and Stark, W. M. (2016) Site-specific recombinases: molecular machines for the Genetic Revolution. Biochem. J. 473, 673-684.

(24) Hirano, N., Muroi, T., Takahashi, H., and Haruki, M. (2011) Site-specific recombinases as tools for heterologous gene integration. Appl. Microbiol. Biotechnol. 92, 227-239.

(25) Santos, C. N. S., Regitsky, D. D., and Yoshikuni, Y. (2013) Implementation of stable and complex biological systems through recombinase-assisted genome engineering. Nat. Commun. 4, 2503.

(26) Wallace, H. A. C., Marques-Kranc, F., Richardson, M., Luna-Crespo, F., Sharpe, J. A., Hughes, J., Wood, W. G., Higgs, D. R., and Smith, A. J. H. (2007) Manipulating the Mouse Genome to Engineer Precise Functional Syntenic Replacements with Human Sequence. Cell 128, 197-209.

(27) Wang, G., Zhao, Z., Ke, J., Engel, Y., Shi, Y.-M., Robinson, D., Bingol, K., Zhang, Z., Bowen, B., Louie, K., Wang, B., Evans, R., Miyamoto, Y., Cheng, K., Kosina, S., De Raad, M., Silva, L., Luhrs, A., Lubbe, A., Hoyt, D. W., Francavilla, C., Otani, H., Deutsch, S., Washton, N. M., Rubin, E. M., Mouncey, N. J., Visel, A., Northen, T., Cheng, J.-F., Bode, H. B., and Yoshikuni, Y. (2019) CRAGE enables rapid activation of biosynthetic gene clusters in undomesticated bacteria. Nat. Microbiol. 4, 2498-2510.

(28) Garcia-Morales, L., Ruiz, E., Gourgues, G., Rideau, F., Piñero-Lambea, C., Lluch-Senar, M., Blanchard, A., and Lartigue, C. (2020) A RAGE Based Strategy for the Genome Engineering of the Human Respiratory Pathogen Mycoplasma pneumoniae. ACS Synth. Biol. 9, 2737-2748.

(29) Baer, A., and Bode, J. (2001) Coping with kinetic and thermodynamic barriers: RMCE, an efficient strategy for the targeted integration of transgenes. Curr. Opin. Biotechnol. 12, 473480.

(30) Forment, J. V., Ramón, D., and MacCabe, A. P. (2006) Consecutive gene deletions in Aspergillus nidulans: Application of the Cre/loxP system. Curr. Genet. 50, 217-224. 
(31) Krappmann, S., Bayram, Ö., and Braus, G. H. (2005) Deletion and allelic exchange of the Aspergillus fumigatus veA locus via a novel recyclable marker module. Eukaryot. Cell 4, 12981307.

(32) Mizutani, O., Masaki, K., Gomi, K., and lefuji, H. (2012) Modified Cre-loxP recombination in Aspergillus oryzae by direct introduction of Cre recombinase for marker gene rescue. Appl. Environ. Microbiol. 78, 4126-4133.

(33) Steiger, M. G., Vitikainen, M., Uskonen, P., Brunner, K., Adam, G., Pakula, T., Penttilä, M., Saloheimo, M., MacH, R. L., and Mach-Aigner, A. R. (2011) Transformation system for Hypocrea jecorina (Trichoderma reesei) that favors homologous integration and employs reusable bidirectionally selectable markers. Appl. Environ. Microbiol. 77, 114-121.

(34) Honda, S., and Selker, E. U. (2009) Tools for Fungal Proteomics: Multifunctional Neurospora Vectors for Gene Replacement, Protein Expression and Protein Purification. Genetics 182, 11-23.

(35) Florea, S., Andreeva, K., Machado, C., Mirabito, P. M., and Schardl, C. L. (2009) Elimination of marker genes from transformed filamentous fungi by unselected transient transfection with a Cre-expressing plasmid. Fungal Genet. Biol. 46, 721-730.

(36) Jiang, B., Zhang, R., Feng, D., Wang, F., Liu, K., Jiang, Y., Niu, K., Yuan, Q., Wang, M., Wang, H., Zhang, Y., and Fang, X. (2016) A tet-on and Cre-loxP based genetic engineering system for convenient recycling of selection markers in Penicillium oxalicum. Front. Microbiol. 7,485 .

(37) Krappmann, S. (2014) Genetic surgery in fungi: Employing site-specific recombinases for genome manipulation. Appl. Microbiol. Biotechnol. 98, 1971-1982.

(38) Roux, I., Bowles, S., Kalaitzis, J. A., Vuong, D., Lacey, E., Chooi, Y.-H., and Piggott, A. M. (2021) Characterisation and heterologous biosynthesis of burnettiene $A$, a new polyenedecalin polyketide from Aspergillus burnettii. Org. Biomol. Chem. 19, 9506-9513.

(39) Lee, G., and Saito, I. (1998) Role of nucleotide sequences of loxP spacer region in Cremediated recombination. Gene 216, 55-65.

(40) Albert, H., Dale, E. C., Lee, E., and Ow, D. W. (1995) Site-specific integration of DNA into wild-type and mutant lox sites placed in the plant genome. Plant J. 7, 649-659.

(41) Chiang, Y. M., Ahuja, M., Oakley, C. E., Entwistle, R., Asokan, A., Zutz, C., Wang, C. C. C., and Oakley, B. R. (2016) Development of Genetic Dereplication Strains in Aspergillus nidulans Results in the Discovery of Aspercryptin. Angew. Chemie - Int. Ed. 55, 1662-1665.

(42) Gilchrist, C. L. M., Lacey, H. J., Vuong, D., Pitt, J. I., Lange, L., Lacey, E., Pilgaard, B., 
Chooi, Y.-H., and Piggott, A. M. (2020) Comprehensive chemotaxonomic and genomic profiling of a biosynthetically talented Australian fungus, Aspergillus burnettii sp. nov. Fungal Genet. Biol. 143, 103435.

(43) Jensen, N. B., Strucko, T., Kildegaard, K. R., David, F., Maury, J., Mortensen, U. H., Forster, J., Nielsen, J., and Borodina, I. (2014) EasyClone: Method for iterative chromosomal integration of multiple genes in Saccharomyces cerevisiae. FEMS Yeast Res. 14, 238-248.

(44) Robellet, X., Oestreicher, N., Guitton, A., and Vélot, C. (2010) Gene silencing of transgenes inserted in the Aspergillus nidulans alcM and/or alcS loci. Curr. Genet. 56, 341348.

(45) Van Leeuwe, T. M., Arentshorst, M., Ernst, T., Alazi, E., Punt, P. J., and Ram, A. F. J. (2019) Efficient marker free CRISPR/Cas9 genome editing for functional analysis of gene families in filamentous fungi. Fungal Biol. Biotechnol. 6, 13.

(46) Mózsik, L., Pohl, C., Meyer, V., Bovenberg, R. A. L., Nygård, Y., and Driessen, A. J. M. (2021) Modular Synthetic Biology Toolkit for Filamentous Fungi. ACS Synth. Biol. 10, 28502861.

(47) Van Dolleweerd, C. J., Kessans, S. A., Van De Bittner, K. C., Bustamante, L. Y., Bundela, R., Scott, B., Nicholson, M. J., and Parker, E. J. (2018) MIDAS: A Modular DNA Assembly System for Synthetic Biology. ACS Synth. Biol. 7, 1018-1029.

(48) Merrick, C. A., Zhao, J., and Rosser, S. J. (2018) Serine Integrases: Advancing Synthetic Biology. ACS Synth. Biol. 7, 299-310.

(49) De Lorenzo, V., Krasnogor, N., and Schmidt, M. (2021) For the sake of the Bioeconomy: define what a Synthetic Biology Chassis is ! N. Biotechnol. 60, 44-51.

(50) Fang, F., Salmon, K., Shen, M. W. Y., Aeling, K. A., Ito, E., Irwin, B., Tran, U. P. C., Hatfield, G. W., Da Silva, N. A., and Sandmeyer, S. (2011) A vector set for systematic metabolic engineering in Saccharomyces cerevisiae. Yeast 28, 123-136.

(51) Lim, F. Y., Sanchez, J. F., Wang, C. C. C., and Keller, N. P. (2012) Toward awakening cryptic secondary metabolite gene clusters in filamentous fungi. Methods Enzymol. 517, 303324. 